\title{
Early growth response 3 inhibits growth of hepatocellular carcinoma cells via upregulation of Fas ligand
}

\author{
SHUJUAN ZHANG ${ }^{1}$, CHAO XIA $^{1}$, CONG XU $^{1}$, JING LIU $^{2}$, HAN ZHU $^{1}$, YA YANG ${ }^{1}$, \\ FEI XU ${ }^{1}$, JUANFENG ZHAO ${ }^{1}$, YING CHANG ${ }^{1}$ and QIU ZHAO $^{1}$ \\ ${ }^{1}$ Department of Gastroenterology and Hepatology, Tongji Hospital, Tongji Medical College, \\ Huazhong University of Science and Technology, Wuhan, Hubei 430030; \\ ${ }^{2}$ Department of Gastroenterology, Zhongnan Hospital, Wuhan University, Wuhan, Hubei 430071, P.R. China
}

Received September 10, 2016; Accepted November 7, 2016

DOI: 10.3892/ijo.2017.3855

\begin{abstract}
Hepatocellular carcinoma (HCC) is a prevalent malignancy with aggressive biological behavior and poor prognosis. Early growth response 3 (EGR3) is a zinc finger transcription factor, and has been studied primarily in the context of neurodevelopment, autoimmunity, inflammation and angiogenesis. Accumulating evidence indicates that EGR3 is a novel suppressor gene of tumor initiation and progression in certain cancer events, but little work has been carried out in exploring the relationship between EGR3 and HCC growth. The purpose of this study was to investigate the possible effects of EGR3 on cell proliferation and apoptosis in HCC, and determine the underlying mechanisms. Here, we observed that EGR3 expression was frequently downregulated in HCC tissues and cell lines. Ectopic expression of EGR3 contributed to cell proliferation inhibition and apoptosis induction in HCC cells in vitro. Furthermore, the expression of Fas ligand (FasL) was significantly enhanced following upregulation of EGR3 in HCC cells, accompanied by an obvious increase of pro-apoptotic Bak and cell cycle inhibitor p21 expression. Based on nude mouse models, we demonstrated that ectopic expression of EGR3 markedly restricted tumor growth, and the expression of FasL was significantly increased in the xenograft tumor tissues which exhibited high EGR3 expression. We further established a co-transfection in HCC cells with EGR3 overexpression plasmid and FasL siRNA. We found that silencing of FasL gene impeded the anti-proliferative and pro-apoptotic effects, as well as the increase of Bak and p21 expression, suggesting an essential role of FasL in EGR3-
\end{abstract}

Correspondence to: Professor Qiu Zhao or Professor Ying Chang, Department of Gastroenterology and Hepatology, Tongji Hospital, Tongji Medical College, Huazhong University of Science and Technology, 1095 Jiefang-Str., Wuhan, Hubei 430030, P.R. China

E-mail: zhaoqiu@medmail.com.cn

E-mail: changying@tjh.tjmu.edu.cn

Key words: early growth response 3, hepatocellular carcinoma, proliferation, apoptosis, Fas ligand mediated growth suppression in HCC cells. Collectively, in conclusion, EGR3 contributes to cell growth inhibition via upregulation of FasL in HCC.

\section{Introduction}

Hepatocellular carcinoma ( $\mathrm{HCC}$ ) is a prevalent malignancy and the third leading cause of tumor-associated mortality worldwide (1). The mainstay of treatment for patients with $\mathrm{HCC}$ at early stage is curative resection. However, despite significant progression in diagnosis of HCC, most HCC patients are diagnosed at an advanced tumor stage when resection is not applicable. Owing to high tumor aggressiveness and poor susceptibility to standard chemotherapy strategy, the overall dismal outcome of HCC has not changed satisfactorily, and the 5-year survival rate is only $7 \%$ (2-4). Hence, further insights into the underlying mechanisms of HCC pathogenesis and identification of potential molecules for HCC treatment are urgently needed.

Early growth response (EGR) proteins are a family of zinc finger transcription factors and induced in various cell types in response to a wide range of internal and external stimuli, such as growth factors, cytokines, hypoxia, injury and stress. The EGR family comprises four members (EGR1, EGR2, EGR3 and EGR4), which share a high degree of homology at their DNA-binding zinc finger domains recognizing a GC-rich fragment in the promoter region of multiple target genes (5-7). EGR proteins are involved in various physiologic and pathologic processes. EGR1 is the most intensively studied protein in EGR family, and implicated in ischemic injury, inflammation, atherosclerosis, and cardiovascular pathogenesis (8). Additionally, EGR1 also exhibits tumorsuppressive activity primarily through induction of tumor cell apoptosis in certain cancer events (9-12). In contrast to EGR1, the function of EGR3 is poorly understood. EGR3 has previously been implicated as a regulator in neurodevelopment, autoimmunity, inflammation, angiogenesis, and cancer (13-17). However, both the expression patterns and functions of EGR3 in human cancers are still controversial. Suzuki et al (18) reported that ectopic expression of EGR3 in breast cancer cells caused enhanced cell invasion in vitro and in vivo. In addition, high expression of EGR3 
was observed and associated with poor prognosis in prostate cancer patients (19). On the contrary, a recent study showed that high-level EGR3 expression in A549 (lung adenocarcinoma) cells resulted in strong inhibition of cell growth, and was prone to show better prognosis in lung adenocarcinoma (20). Furthermore, EGR3 was a potent limiting factor for the proliferative potential of hematopoietic stem cells (HSCs) in leukemia (21). Downregulation of EGR3 was visible and related to adverse outcome in gastric cancer (22).

The relationship between EGR3 and HCC growth has not yet been elucidated. To address this question, we observed the expression pattern in human HCC specimens, human normal hepatic and HCC cell lines. Furthermore, we evaluated the effect of EGR3 on the growth of HCC cells and explored the underlying mechanisms.

Fas ligand (FasL), a 40-kDa glycosylated type 2 transmembrane protein, is a member of the tumor necrosis factor (TNF) family (23). Binding of FasL to its surface receptor, Fas, leads to an adaptor molecule, known as Fas-associated death domain (FADD), recruitment to the cytoplasmic domain of this death receptor, and activates caspase- 8 . Then activated caspase- 8 triggers the activation of several downstream caspase substrates and apoptosis is ultimately induced in target cells $(24,25)$. In contrast to the ubiquitous Fas protein, FasL is concentrated in activated $\mathrm{T}$ lymphocytes and natural killer (NK) cells, as well as in some non-lymphoid tissues, such as eyes, testis, trophoblasts and cancer cells (26-30). Given its role in apoptosis inhibition, FasL has been studied extensively in tumor field for the past several years. In a previous study, high-level FasL expression caused significant inhibition of cell growth in SGC-7901 cells (human gastric cancer cells) (31). In addition, FasL has been reported to play an essential role in gemcitabine-mediated cell death in non-small cell lung cancer (NSCLC) cells (32). Bianco et al (33) performed intratumoral injection of a plasmid encoding human FasL into oral malignant melanoma in dogs to test FasL-based gene therapy. They found that three out of five dogs displayed tumor volume reduction, providing confirmation of the pro-apoptotic effect of FasL gene in vivo. These results suggest therapeutic potential of FasL for certain types of cancers.

It is well established that the promoter region of FasL gene possesses multiple sites of DNA-protein interaction, which can be identified by members of some transcription factor families, such as nuclear factor in activated T cells (NFAT), EGR and nuclear factor-kappa $\mathrm{B}(\mathrm{NF}-\kappa \mathrm{B})$ families, leading to increased expression of FasL (34). Some studies have described a key role of EGR3 in triggering transactivation of the FasL promoter in T cells, HeLa cells and MCF-7 cells (breast cancer cells) $(35,36)$. However, little is known about the relationship between EGR3 and FasL in HCC, and the combined action of EGR3 and FasL on the growth of cancer cells is even less documented.

In this study, we confirm that EGR3 is frequently downregulated in human HCC tissues and cell lines, and high-level expression of EGR3 exhibits significant growth suppression of HCC cells both in vitro and in vivo. Furthermore, our results indicate for the first time that the close cooperation between EGR3 and FasL is a novel regulator mechanism of the growth of HCC cells.

\section{Materials and methods}

Cell culture and HCC specimens. The human HCC cell lines (PLC/PRF/5, HCC-LM3, Huh7, HepG2) and human normal hepatic cell line L02 were obtained from Institute of Liver Diseases, Tongji Hospital of Tongji Medical College, Huazhong University of Science and Technology (HUST, Wuhan, China). All the cells were routinely cultured in Dulbecco's modified Eagle's medium (DMEM; Gibco, Carlsbad, CA, USA) supplemented with $10 \%$ fetal bovine serum (FBS; Gibco, Grand Island, NY, USA) and maintained in an incubator with $5 \% \mathrm{CO}_{2}$ at $37^{\circ} \mathrm{C}$. After approval by the Ethics Committee of Tongji Hospital of HUST and obtaining the informed consent from all tissue donors, specimens (including 25 pairs of fresh HCC and adjacent non-tumor tissues) were collected at Tongji Hospital and stored in liquid nitrogen immediately after surgery until RNA and protein extraction.

RNA isolation and quantitative real-time PCR ( $q R T-P C R)$. Total RNA was extracted from tissues or cells using TRIzol reagent (Invitrogen, Carlsbad, CA, USA) according to the manufacturer's instructions. After quantification by NanoDrop 2000 (Thermo Scientific, Wilmington, DE, USA), $500 \mathrm{ng}$ of total RNA was reversely transcribed into cDNA by a PrimeScript RT Reagent kit (Takara, Tokyo, Japan). Briefly, each RNA sample was added to the reaction mixture comprising 5X PrimeScript RT Master Mix and nuclease-free water, followed by incubation at $37^{\circ} \mathrm{C}$ for $15 \mathrm{~min}$ and $85^{\circ} \mathrm{C}$ for 5 sec. Then, the target genes were amplified in $10 \mu \mathrm{l}$ of PCR reaction solution composed of cDNA, primers, nuclease-free water and SYBR Premix Ex Taq (Takara) in a StepOne RealTime PCR system (Applied Biosystem, Carlsbad, CA, USA). The PCR amplification was conducted at $95^{\circ} \mathrm{C}$ for $30 \mathrm{sec}$ followed by 40 cycles of $5 \mathrm{sec}$ at $95^{\circ} \mathrm{C}$ and $30 \mathrm{sec}$ at $60^{\circ} \mathrm{C}$. GAPDH was used as an internal control to normalize gene expression, and relative quantification was performed using the $2^{-\Delta \Delta \mathrm{CT}}$ method.

Primers used in this study were as follows: EGR3 sense, 5'-GACATCGGTCTGACCAACGAG-3'; antisense, 5'-GGCG AACTTTCCCAAGTAGGT-3'. FasL sense, 5'-TGCCTTGGT AGGATTGGGC-3', antisense, 5'-GCTGGTAGACTCTCGGA GTTC-3'. Bak sense, 5'-ATGGTCACCTTACCTCTGCAA-3'; antisense, 5'-TCATAGCGTCGGTTGATGTCG-3'. p21 sense, 5'-TGTCCGTCAGAACCCATGC-3'; antisense, 5'-AAAGTCG AAGTTCCATCGCTC-3'. GAPDH sense, 5'-GGGAAGCTT GTCATCAATGG-3'; antisense, 5'-CATCGCCCCACTTG ATTTTG-3'.

Western blot analysis. Tissues or cells were lysed in ice-cold RIPA lysis buffer (Beyotime Biotechnology, Shanghai, China) with a protease inhibitor cocktail (Roche Diagnostics, Indianapolis, IN, USA) for $30 \mathrm{~min}$ on ice. After centrifugation at $12,000 \mathrm{x}$ for $10 \mathrm{~min}$ at $4^{\circ} \mathrm{C}$, the supernatant of lysis was collected and quantified by a BCA Protein Assay kit (Pierce Biotechnology, Rockford, IL, USA) according to the manufacturer's instructions. Equal amounts of protein $(40 \mu \mathrm{g})$ were separated by 10 or $12 \%$ sodium dodecyl sulfate-polyacrylamide gel electrophoresis (SDS-PAGE) and then transferred onto polyvinylidene difluoride (PVDF) membranes (Millipore, Bedford, MA, USA). After blocking with $5 \%$ bovine serum 
albumin (BSA; Sigma, St. Louis, MO, USA) for $1 \mathrm{~h}$ at room temperature, membranes were probed with primary antibodies against EGR3 (sc-191, Santa Cruz, CA, USA; 1:500 dilution), FasL (ab68338, Abcam, Cambridge, UK; 1:500 dilution), Bak (\#6947, Cell Signaling Technology, Danvers, MA, USA; 1:1,000 dilution), p21 (\#2947, Cell Signaling Technology; 1:1,000 dilution) and GAPDH (10494-1-AP, Proteintech Group, Chicago, IL, USA; 1:5,000 dilution) at $4^{\circ} \mathrm{C}$ overnight. The membranes were subsequently rinsed and further incubated with a horseradish peroxidase (HRP)-conjugated goat antirabbit secondary antibody (A21020, Abbkine, Redlands, CA, USA; 1:4,000 dilution) for $2 \mathrm{~h}$, followed by visualization with enhanced chemiluminescence reagent (Pierce Biotechnology). GAPDH served as an endogenous control to confirm equal loading of proteins.

Plasmid, small interfering RNA (siRNA) and transfection. The human overexpression plasmid pGV219-EGR3 and its control plasmid pGV219-Vector were purchased from Genechem Co., Ltd. (Shanghai, China). The FasL siRNA (si-FasL) and non-specific siRNA (si-NC) were designed and synthesized by RiboBio Co., Ltd. (Guangzhou, China). The effective sequence of siRNA targeting FasL gene was 5'-GCAAGTCCAACTCAAGGTC-3'. Transient transfection was performed using the Lipofectamine 2000 reagent (Invitrogen) according to the manufacturer's instructions. At the indicated times after transfection, cells were subjected to further experiments.

CCK-8 assay. Cell proliferation was assessed using Cell Counting Kit-8 (CCK-8) (Dojindo Laboratories, Kumamoto, Japan). After trypsinization and resuspension in culture medium, Huh7 and HCC-LM3 cells were seeded into 96-well plates at $8 \times 10^{3}$ cells/well. At $80-90 \%$ confluency, cells were subjected to transient transfection, and subsequently cultured for 24,48 or $72 \mathrm{~h}$. Then, $10 \mu \mathrm{l}$ of CCK- 8 solution was added to each well followed by incubation at $37^{\circ} \mathrm{C}$ for $2 \mathrm{~h}$, and optical density (OD) values were detected at a wavelength of $450 \mathrm{~nm}$ with a BioTek ELX800 microplate reader (BioTek, Vermont, NE, USA). At least six wells were used for each group, and wells with cell culture medium of the same volume only served as blanks.

Colony formation assay. At $24 \mathrm{~h}$ post-transfection, cells were harvested and seeded into fresh 6 -well plates ( $1 \times 10^{3}$ cells/well). After culture for 2 weeks, the formed colonies were fixed with $4 \%$ paraformaldehyde and stained using $0.1 \%$ crystal violet to visualize colonies for quantification. Percentage of colony formation was calculated by the following formula: colony formation rate $(\%)=$ numbers of colonies/numbers of seeded cells $\mathrm{x} 100 \%$.

Apoptosis analysis. Cell apoptosis was quantitatively determined by flow cytometry with Annexin V-PE/7-AAD Apoptosis Detection kit (BD Biosciences, San Diego, CA, USA) according to the manufacturer's instructions. Briefly, at $48 \mathrm{~h}$ post-transfection, cells were collected, washed twice with cold PBS, and resuspended in $1 \mathrm{X}$ binding buffer (1x $10^{6}$ cells $\left./ \mathrm{ml}\right)$. PE-conjugated Annexin V $(5 \mu \mathrm{l})$ and $5 \mu \mathrm{l}$ of 7-amino-actinomycin D (7-AAD) were subsequently added to $100 \mu \mathrm{l}$ of cell preparations $\left(1 \times 10^{5}\right.$ cells $)$ and incubated for $15 \mathrm{~min}$ at room temperature in the dark. After supplementation with further $400 \mu \mathrm{l}$ of $1 \mathrm{X}$ binding buffer to each sample, the cells were analyzed by a FACSCalibur instrument (BD Biosciences) within $1 \mathrm{~h}$.

Lentivirus-based EGR3 overexpression. To facilitate in vivo observation of EGR3 function, we commissioned Genechem Co., Ltd. to constructed a recombinant lentivirus LV-EGR3 and its control LV-Vector, and transduced them to Huh7 cells according to the manufacturer's instructions. Briefly, Huh7 cells in good condition were seeded into $10-\mathrm{cm}$ culture dishes. When reaching 30-40\% confluency, cells were transduced with LV-EGR3 or LV-Vector, respectively. Twenty-four hours later, cells were washed and covered by fresh DMEM with $10 \%$ FBS. After 48 h, $2 \mu \mathrm{g} / \mathrm{ml}$ puromycin (Sigma) was applied to screen out the uninfected cells. Once LV-EGR3 or LV-Vector transfected Huh7 cells were established successfully, they were used for in vivo tumor xenograft experiment.

Xenograft tumor models. Twelve BALB/c nude mice (female, 4-6-week-old, 16-20 g) were purchased from HFK Bioscience Co., Ltd. (Beijing, China). The mice were randomly divided into two groups ( $n=6$ in each group) and bred in the SPF Animal Institute of Tongji Medical College. All animal experiments were carried out according to the Tongji Medical College Institutional Animal Care and Use Committee Guidelines. The LV-EGR3 or LV-Vector transfected Huh7 cells were harvested from tissue culture dishes and washed twice with PBS. Then cells $\left(2 \times 10^{7}\right.$ cells $\left./ \mathrm{ml}\right)$ were resuspended in Matrigel (BD Biosciences) diluted with serum-free DMEM at the ratio of $1: 1$, and injected subcutaneously $\left(2 \times 10^{6}\right.$ cells per mouse) into the flank of each mouse. After 10 days, the length (L) and width (W) of the tumors were measured externally using a digital vernier caliper every 4 days. Tumor volume was determined according to the formula: $\mathrm{V}=\left(\mathrm{Lx} \mathrm{W}^{2}\right) / 2$. At the termination of the experiment, mice were sacrificed and tumor tissue from each mouse was excised, photographed and weighed.

Statistics. Statistical analysis was carried out by SPSS 21.0 software (SPSS Inc., Chicago, IL, USA). All the data are presented as mean \pm standard deviation (SD). Statistical differences between groups and among groups were compared using Student's t-test and ANOVA, respectively. A value of $\mathrm{P}<0.05$ was considered to indicate a statistically significant difference. Each experiment was independently performed at least three times.

\section{Results}

Expression of EGR3 is frequently downregulated in human HCC specimens and cell lines. To evaluate the possible relationship between EGR3 and HCC, EGR3 expression pattern was analyzed in a set of 25 human HCC specimens, four different human HCC cell lines (PLC/PRF/5, HCC-LM3, Huh7 and HepG2), and human normal hepatic cell line L02. As shown in Fig. 1A, 23 out of 25 cases exhibited lower EGR3 transcripts in HCC tissues than that in matched adjacent non-tumor tissues $(\mathrm{P}<0.05)$. Subsequently, the protein levels 
A

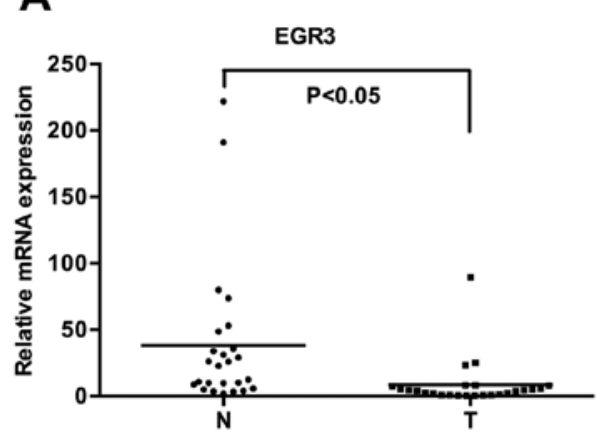

C

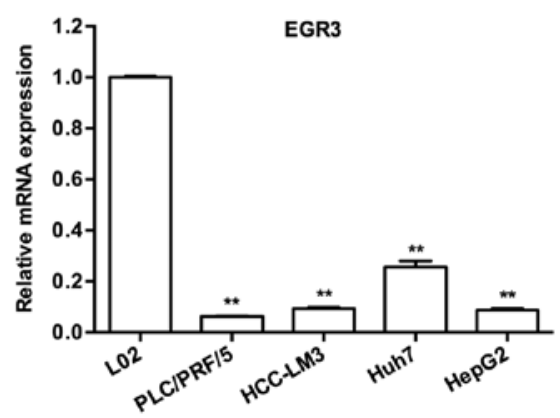

B $\frac{1}{N T} \frac{2}{N T} \frac{3}{N T} \frac{4}{N T} \frac{5}{N T} \frac{6}{N T}$

EGR3

GAPDH

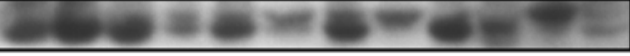

$\frac{7}{N T} \frac{8}{N T} \frac{9}{N T} \frac{10}{N T} \frac{11}{N T} \frac{12}{N T}$

EGR3

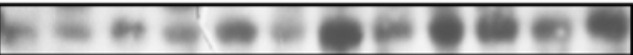

GAPDH

D

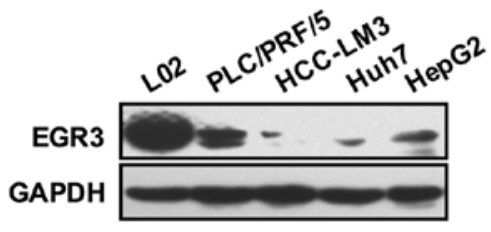

Figure 1. EGR3 is frequently downregulated in HCC tissues and cell lines. (A) The mRNA levels of EGR3 in 25 pairs HCC and their matched adjacent non-tumor tissues were measured by qRT-PCR. (B) Representative images showed EGR3 protein expression in partial pairs (n=12) HCC and the corresponding adjacent non-tumor tissues. Differential expression of EGR3 in four human HCC cell lines (PLC/PRF/5, HCC-LM3, Huh7 and HepG2) and the normal hepatic cell line L02 by qRT-PCR (C) and western blot analysis (D), respectively. Data are presented as the mean $\pm \mathrm{SD} .{ }^{* * *} \mathrm{P}<0.01$. T, tumor tissues; $\mathrm{N}$, non-tumor tissues.

A

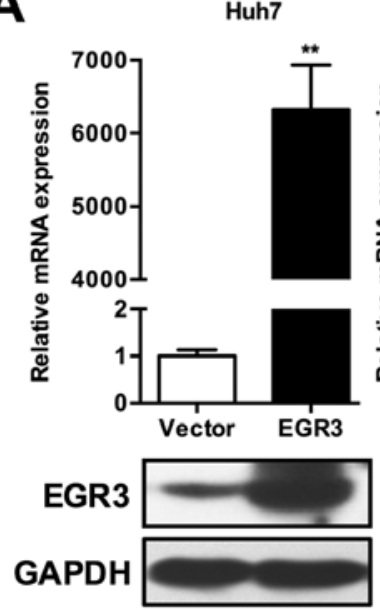

HCC-LM3

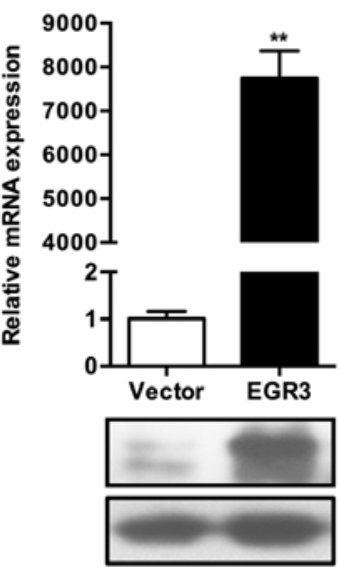

B

C

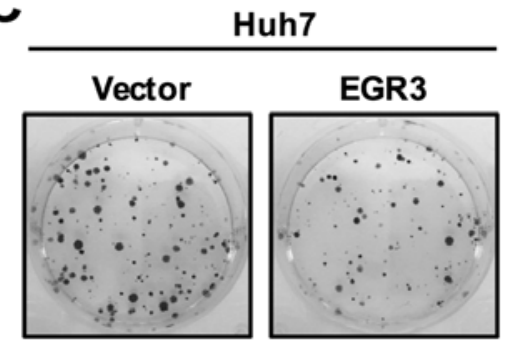

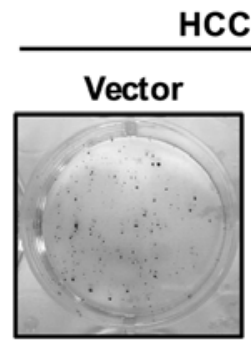
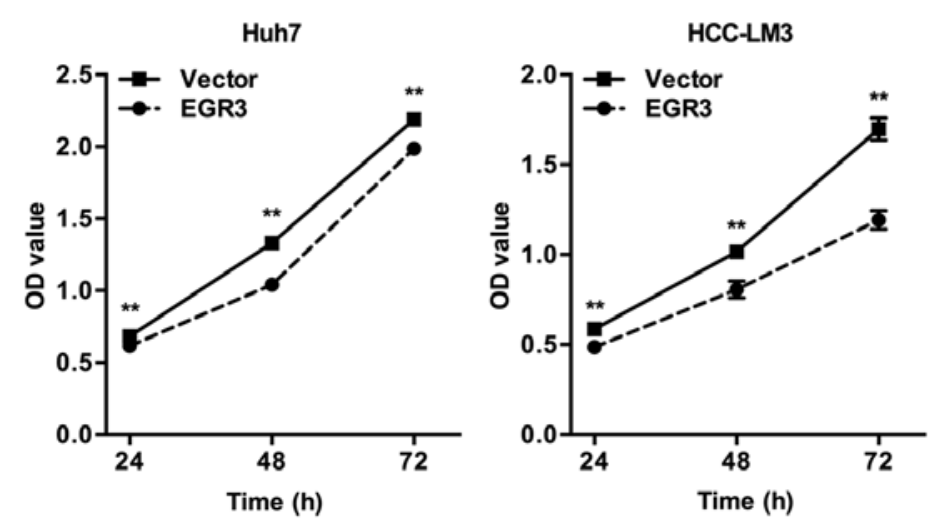

Figure 2. EGR3 has a strong tumor suppressive effect on HCC cell proliferation in vitro. (A) Relative expression of EGR3 in Huh7 and HCC-LM3 cells transfected with EGR3 plasmid or vector control plasmid was examined by qRT-PCR and western blot analysis, respectively. (B) OD values of transfected Huh7 and HCC-LM3 cells were detected by Cell Counting Kit-8 (CCK-8) at various time-points (24, 48 and 72 h). (C) Representative images (left) and quantification graphs (right) of crystal violet-stained cell colonies in transfected Huh7 and HCC-LM3 cells. Data are presented as the mean \pm SD of three independent experiments. ${ }^{*} \mathrm{P}<0.05,{ }^{* *} \mathrm{P}<0.01$. 
A

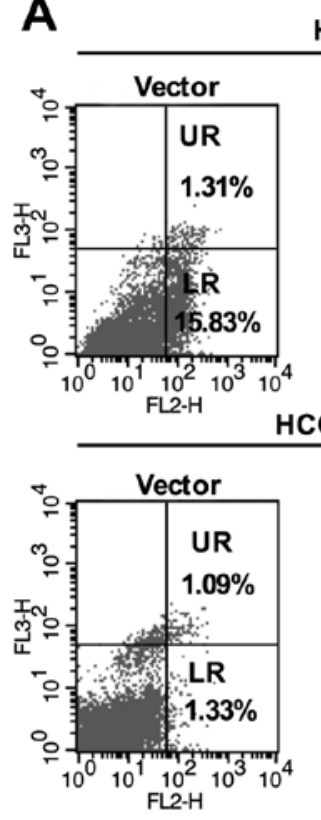

Huh7
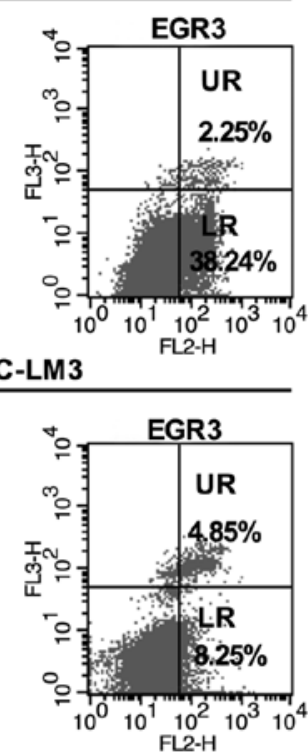

B

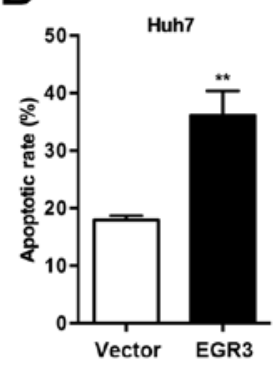

D

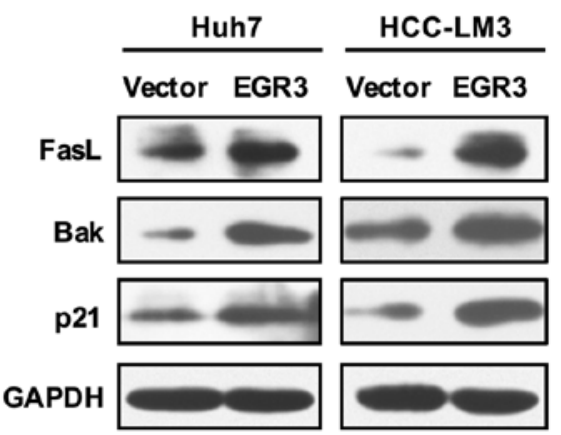

C
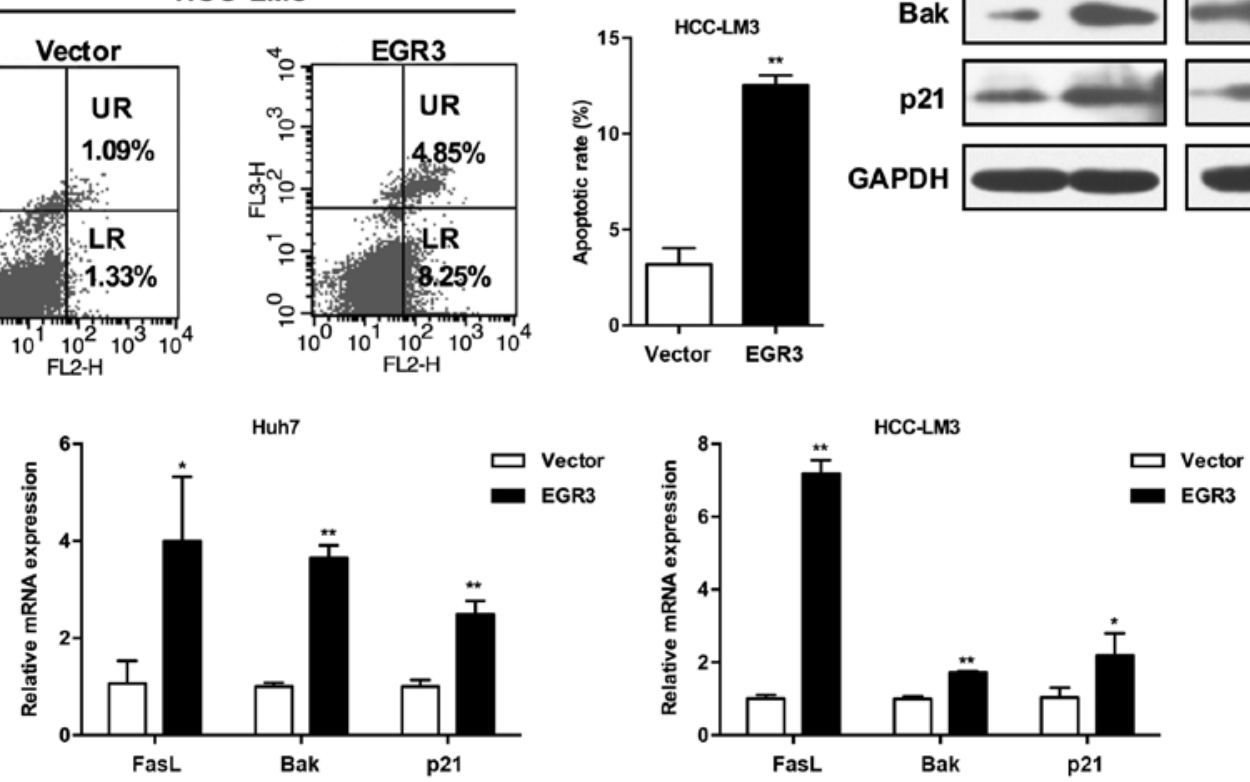

Figure 3. EGR3 induces apoptosis and upregulates expression of FasL, Bak, and p21 in HCC cells in vitro. Flow cytometric analysis was used to quantify apoptosis induced by EGR3. Representative FACS histograms (A) and quantification bar graphs (B) of total apoptotic cells in transfected Huh7 and HCC-LM3 cells at $48 \mathrm{~h}$ post-transfection. The relative expression of FasL, Bak and p21 in transfected Huh7 and HCC-LM3 cells was confirmed by qRT-PCR (C) and western blot analysis (D), respectively. Data are presented as the mean $\pm \mathrm{SD}$ of three independent experiments. ${ }^{*} \mathrm{P}<0.05{ }^{* * *} \mathrm{P}<0.01$.

of EGR3 were determined by western blot analysis, and they were frequently downregulated in HCC tissues as we expected (Fig. 1B showed protein bands of EGR3 of partial specimens). Similarly, a remarkable decrease in the expression of EGR3 mRNA and protein was also observed in four HCC cell lines compared with the normal hepatic cell line L02 [Fig. 1C and $\mathrm{D} ; \mathrm{P}<0.01]$. These findings revealed a potential association between low EGR3 expression and HCC pathogenesis.

EGR3 inhibits HCC cell proliferation. To explore the potential role of EGR3 in HCC, successful overexpression of EGR3 in Huh7 and HCC-LM3 cells were first assessed by qRT-PCR and western blot analysis (Fig. 2A). CCK-8 and colony formation assays were subsequently performed to assess the effect of ectopic EGR3 expression on proliferation in Huh7 and HCC-LM3 cells. As demonstrated in Fig. 2B, CCK-8 analysis in two HCC cell lines showed that OD values of EGR3 overexpressing cells at various time-points $(24,48$ and $72 \mathrm{~h})$ were remarkably lower than that of the control cells $(\mathrm{P}<0.01)$. In colony formation assay, high-level expression of EGR3 caused significant decline of colony-forming ability as evidenced by the obvious decrease of colony formation rates compared with the control cells. The colony formation rates of Huh7 cells in

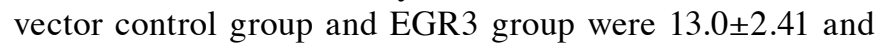

$9.3 \pm 0.82 \%$, respectively (Fig. $2 \mathrm{C} ; \mathrm{P}<0.05$ ). Similar result of colony formation assay was also observed in HCC-LM3 cells, as summarized in Fig. 2C (vector control group and EGR3 group, 9.9 \pm 2.36 and $5.2 \pm 1.46 \%$, respectively; $\mathrm{P}<0.05)$. These data indicated the effect of EGR3 on proliferation inhibition in HCC cells.

EGR3 induces HCC cell apoptosis. The flow cytometry was performed to evaluate the involvement of EGR3 in cell apoptosis. Apoptotic cell death was determined by detecting the cells in the lower right (LR) and upper right (UR) quadrants of the graphs (Fig. 3A), which are regarded as early-stage and late-stage apoptotic cells, respectively. As shown in Fig. 3B, EGR3 significantly induced apoptosis in the two HCC cell sections. The percentages of total apoptotic cells (LR+UR quadrants) in vector control group and EGR3 group were as follows: $17.93 \pm 0.78$ and $36.21 \pm 4.14 \%$ in Huh7 cells; $3.18 \pm 0.85$ and $12.52 \pm 0.53 \%$ in HCC-LM3 cells $(\mathrm{P}<0.01)$. These results indicated that EGR3 inhibited growth of HCC cells partially through the induction of apoptosis.

EGR3 upregulates expression of FasL, Bak, and p21 in HCC cells. Given the previous studies that EGR3 promoted transactivation of FasL gene in certain cancer cells, we speculated 
A

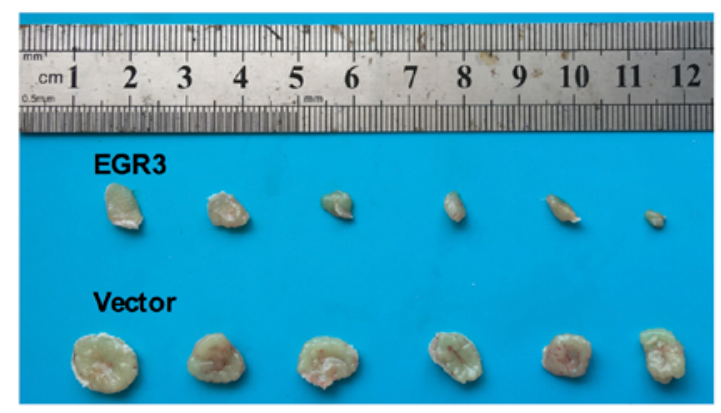

C

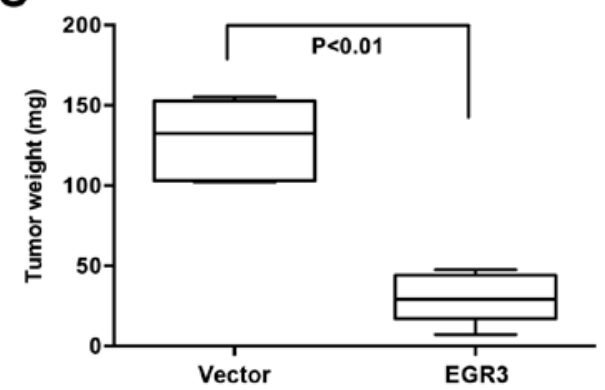

E

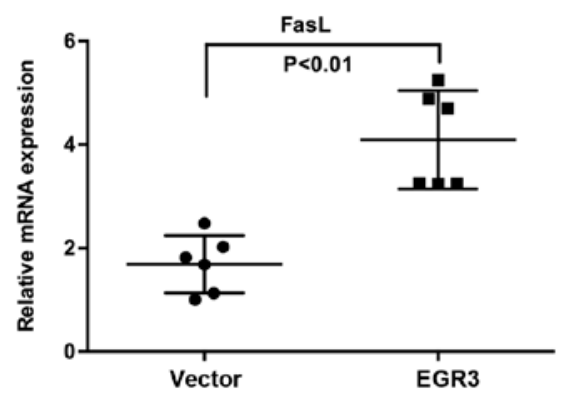

B

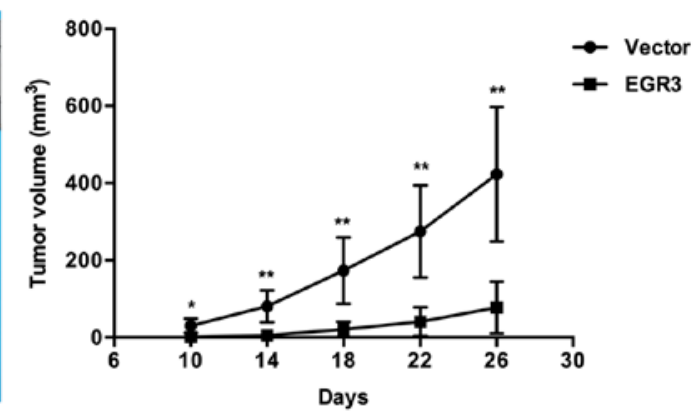

D

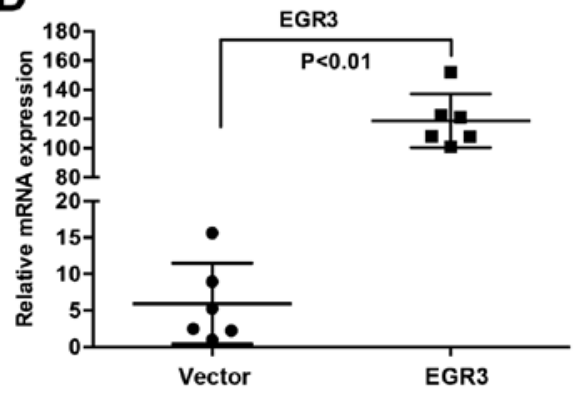

$\mathbf{F}$

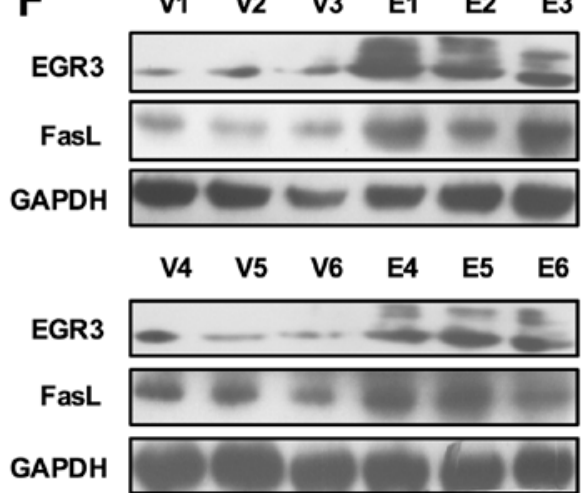

Figure 4. EGR3 restricts tumor growth and increases FasL expression in xenograft mouse models of HCC. (A) Image of isolated tumors from injected mice. (B) At 10 days after tumor cell implantation, growth curves were ploted by measurement of tumor volumes every 4 days with a total period of 26 days. (C) Weights of the isolated tumors. The expression levels of EGR3 and FasL were quantitated by qRT-PCR (D and E) and western blot analysis (F) in subcutaneous xenograft tumors of HCC in nude mice. Data are presented as the mean \pm SD of six samples.

that EGR3 also enhanced FasL expression in HCC cells. Indeed, we found that FasL mRNA and protein expression was elevated simultaneously with increased expression of EGR3 when EGR3 was overexpressed in Huh7 and HCC-LM3 cells (Fig. $3 \mathrm{C}$ and $\mathrm{D} ; \mathrm{P}<0.05, \mathrm{P}<0.01$ ). Furthermore, the expression of pro-apoptotic Bak and cell cycle inhibitor p21 was also detected. Consistent with the changes of FasL expression, both mRNA and protein levels of Bak and p21 were significantly promoted in EGR3 overexpressing cells compared with the control cells (Fig. 3C and D; $\mathrm{P}<0.05, \mathrm{P}<0.01$ ). These results suggested that cell growth inhibition induced by EGR3 was associated with upregulation of FasL, Bak and p21.

EGR3 restricts tumor growth and increases FasL expression in xenograft mouse models of HCC. Following in vitro studies, we sought to determine whether EGR3 restricts in vivo tumor growth by utilizing xenograft mouse models. In view of the identical inhibitory effects of EGR3 on Huh7 and HCC-LM3 cell growth, we selected Huh7 cells for further in vivo studies. At the outset of the experiment, a recombinant lentivirus LV-EGR3 and its control LV-Vector were constructed to transduce Huh7 cells. Once LV-EGR3-Huh7 and LV-Vector-Huh7 cells were established successfully, they were subcutaneously injected into nude mice, and tumor growth was monitored regularly. Consistent with our in vitro results, ectopic expression of EGR3 in Huh7 cells displayed significantly lower tumorigenicity as evidenced by the much smaller volumes of tumor masses isolated from nude mice compared with the control cells (Fig. 4A). Furthermore, a significant retardation of tumor growth and decreased tumor weights were observed in the LV-EGR3-Huh7 group when compared with the LV-Vector-Huh7 group (Fig. 4B and C; $\mathrm{P}<0.05$, $\mathrm{P}<0.01)$. Additionally, we also confirmed that the expression of EGR3 was indeed upregulated in the LV-EGR3-Huh7 

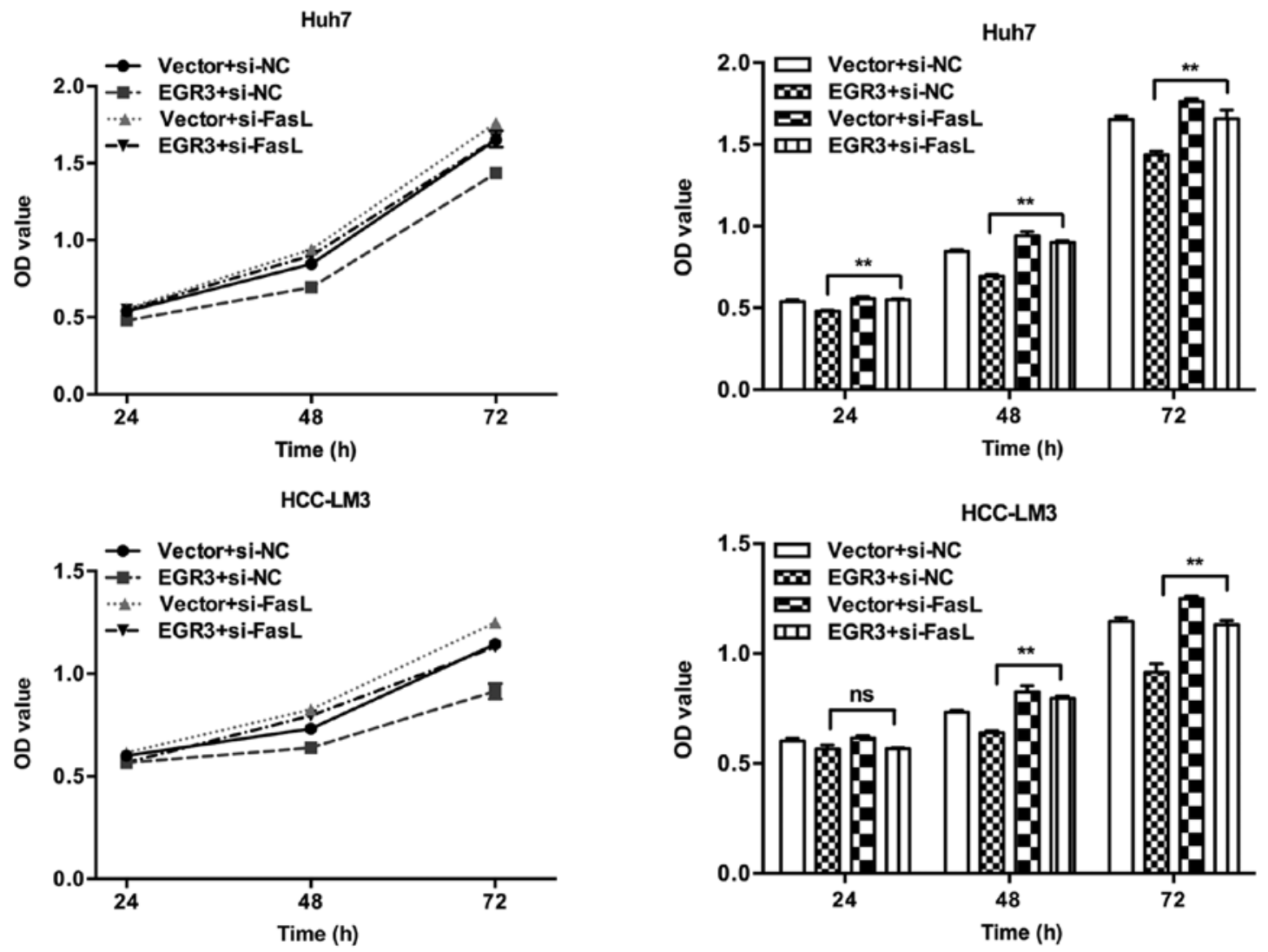

Figure 5. FasL is essential for the suppression of HCC cell growth induced by EGR3. We established a co-transfection in Huh7 and HCC-LM3 cells with EGR3 overexpression plasmid and FasL siRNA. OD values were detected by CCK-8 assay at various time-points (24, 48 and $72 \mathrm{~h})$ after transfection. Data are presented as the mean $\pm \mathrm{SD}$ of three independent experiments. ${ }^{* *} \mathrm{P}<0.01 ; \mathrm{ns}$, no statistical significance.

group compared to that in the LV-Vector-Huh7 group, and the expression of FasL was markedly increased in the xenograft tumor tissues which exhibited high EGR3 expression (Fig. 4D-F; $\mathrm{P}<0.01$ ). These data provided strong evidence that EGR3 restricted tumor growth and promoted FasL expression in vivo.

FasL is essential for the growth suppression induced by EGR3 in HCC cells. To assess the role of FasL in EGR3-mediated growth suppression of HCC cells, we established a co-transfection in Huh7 and HCC-LM3 cells with EGR3 overexpression plasmid and FasL siRNA. CCK8 analysis showed that siRNAmediated silencing of FasL gene significantly attenuated the anti-proliferation effect conferred by EGR3 overexpression in Huh7 and HCC-LM3 cells (Fig. 5; P<0.01). In addition, EGR3induced cell apoptosis was also hampered after silencing of FasL expression $(65.61 \pm 0.72$ and $73.66 \pm 1.02 \%$ in Huh7 cells; $31.46 \pm 1.13$ and $36.88 \pm 0.58 \%$ in HCC-LM3 cells, Fig. 6A; $\mathrm{P}<0.01)$. Finally, we found that knockdown of FasL gene prevented the increase of Bak and p21 expression induced by EGR3 overexpression as well (Fig. 6B). Taken together, these results suggested the essential role of FasL in cell growth inhibition mediated by EGR3 in HCC.

\section{Discussion}

HCC is one of the most common malignancies worldwide and highly prevalent in China. In addition to chronic viral infections and hepatotoxic agents, the pathogenesis of $\mathrm{HCC}$ is associated with abnormalities of several oncogenes and tumor suppressor genes $(37,38)$. A series of studies have been carried out to identify potential molecules for treatment of HCC to date.

EGR3 is a zinc finger transcription factor, which was previously reported to be implicated in neurodevelopment, autoimmunity, inflammation and angiogenesis (13-16). Furthermore, the effects of EGR3 on tumor initiation and progression in certain cancers have also been evaluated, and some studies have described novel role of EGR3 gene as a tumor suppressor in lung carcinoma, leukemia and gastric cancer (20-22). However, the relationship between EGR3 and $\mathrm{HCC}$ has not yet been elucidated. In this study, we assessed the effect of EGR3 on HCC cell growth and investigated the possible molecular mechanisms. We examined the expression pattern of EGR3 in human HCC specimens, human normal hepatic and HCC cell lines. We observed that expression of EGR3 was frequently downregulated in human HCC tissues and cell lines, suggesting a potential association between the low expression of EGR3 and HCC pathogenesis. Subsequently, we utilized gain-of-function approach to determine the effect of EGR3 on cell growth in two HCC cell lines, Huh7 and HCC-LM3 cells. We demonstrated that ectopic expression of EGR3 inhibited proliferation and induced apoptosis, leading to cell growth suppression in Huh7 and HCC-LM3 cells in vitro. Our in vivo results were further confirmed by xenograft mouse models. Lentivirus-mediated EGR3 overexpression in Huh7 
A

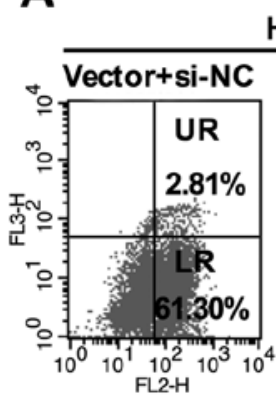

Huh7
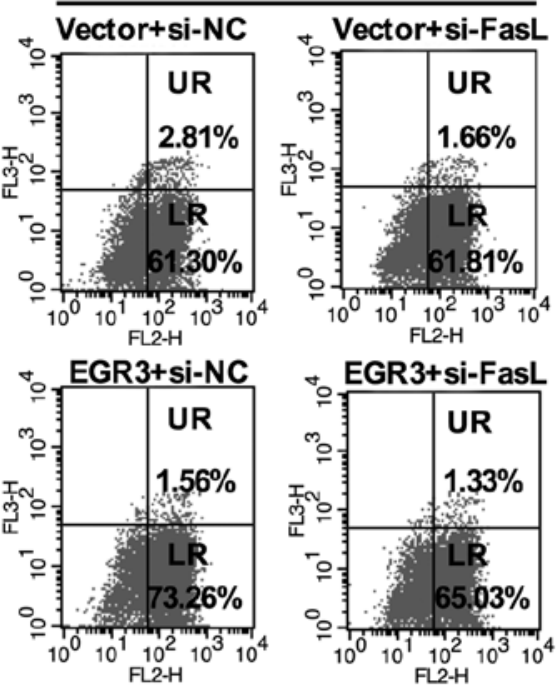

EGR3+si-FasL

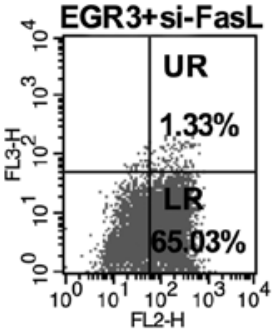

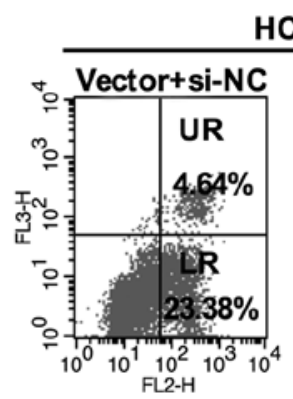

HCC-LM3
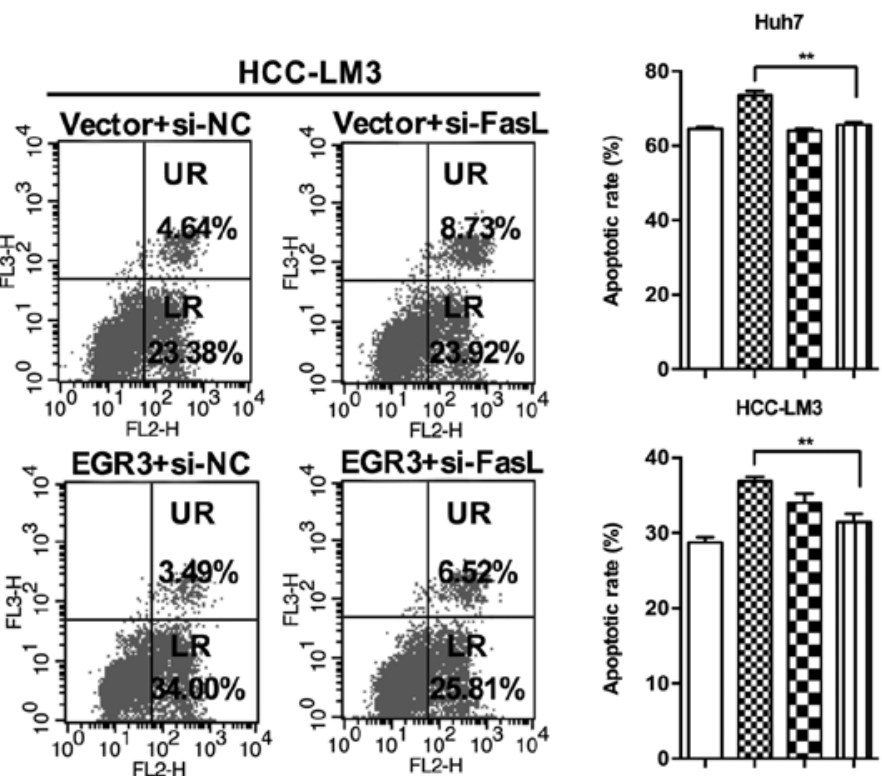

$\square$ Vector+si-NC

EGR3+si-NC

Vectortsi-FasL

m EGR3+si-FasL
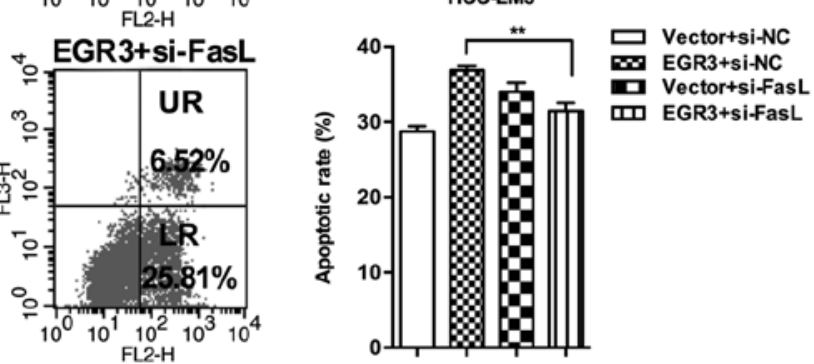

B

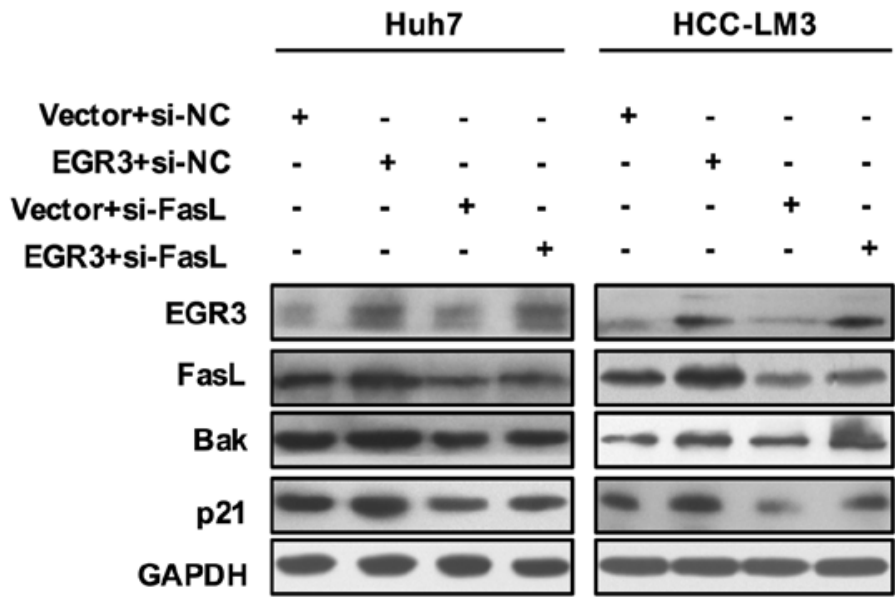

Figure 6. FasL is essential for the suppression of HCC cell growth induced by EGR3. (A) Apoptotic cell percentage was assessed by flow cytometry at $48 \mathrm{~h}$ post-transfection. (B) The protein expression of EGR3, FasL, Bak and p21 was verified by western blot analysis. Data are represented as the mean \pm SD of three independent experiments. ${ }^{* *} \mathrm{P}<0.01$.

cells exhibited obviously lower tumorigenicity as evidenced by the significant retardation of tumor growth, reduced tumor volumes and decreased tumor weights. As a whole, these results provided strong evidence that EGR3 inhibited the growth of HCC cells both in vitro and in vivo, suggesting the potential of EGR3 as a tumor suppressor gene for prevention or treatment of HCC.

Apoptosis is an essential cellular activity involved in multiple physiological and pathological processes. Failure of apoptosis could allow the survival of abnormal cells, which are closely related to tumorigenesis. It is well established that apoptosis can be triggered via two distinct but convergent pathways known as Fas receptor-mediated pathway and mitochondrial pathway, respectively (39). The Fas/FasL apoptotic pathway has been considered a critical mechanism for eliminating tumor cells. It was previously demonstrated that FasL was required for curcumin-induced apoptosis in Huh7 cells (40), and the Fas/FasL pathway contributed to the antitumor effects of a combination therapy of interferon (IFN) $\alpha$ and 5-fluorouracil (5-FU) against HCC cells (41), and intratumoral injections of an adenovirus expressing FasL gene into the HNSCC (head and neck squamous cell carcinoma) cell xenografts induced significant growth suppression of tumors (42). Combining these reports and our experimental results, as well as the role of EGR3 in transactivation of the FasL promoter in certain cell types $(35,36)$, we speculate that EGR3 may also promote FasL expression, and FasL is related to the EGR3mediated cell growth inhibition in HCC cells. Indeed, we observed a remarkable increase of FasL mRNA and protein expression in EGR3 overexpressed Huh7 and HCC-LM3 cells. Furthermore, the phenomenon of high-level FasL expression also existed in xenograft tumor tissues which exhibited high EGR3 expression. In addition, our results revealed that the expression of Bak and p21 was significantly enhanced following upregulation of EGR3 in HCC cells. p21 is a potent inhibitor of cyclin-dependent kinases (CDKs) and inhibits the activity of CDKs following binding to them, leading to cell growth arrest at specific stages of the cell cycle (43). Bak is a pro-apoptotic and acts as a critical downstream effector in apoptosis process to permeabilize the outer mitochondrial membrane, leading to 
the release of apoptogenic factors including cytochrome $c$ to the cytoplasm (44). These results indicated that Bak and p21 were also involved in EGR3-induced cell growth suppression.

Finally, we sought to determine whether FasL is essential for the role of EGR3 in HCC cells in vitro. We established a co-transfection in Huh7 and HCC-LM3 cells with EGR3 overexpression plasmid and FasL siRNA. We found that siRNA-mediated silencing of FasL gene significantly attenuated the effects of anti-proliferation and apoptosis conferred by EGR3 overexpression in Huh7 and HCC-LM3 cells, suggesting an essential role of FasL in EGR3-mediated growth suppression in HCC cells as expect. Furthermore, knockdown of FasL also impeded the increase of Bak and p21 expression induced by EGR3 overexpression. We asked why this phenomenon appeared. Through consulting literature materials, we found that binding of FasL with Fas could activate mitogen-activated protein kinase kinase kinase 5 (ASK1) via death domain-associated protein Daxx (45). The activated ASK1 itself triggers the activation of JNK and p38 by phosphorylation cascades involving a series of protein kinases $(46,47)$. JNK and p38 pathways are key mechanisms involved in stability and induction of p53, and induced apoptosis by activation of p53 in lung carcinoma cells, hepatoma cells, and leukemia cells (48). The p53 tumor suppressor is a potent transcription factor that activates multiple genes in charge of apoptosis, cell cycle arrest, and autophagy (49). Bak and p21 are common transcriptional targets of p53 $(50,51)$. Based on the above studies, we speculate that overexpression of EGR3 can upregulate FasL expression followed by activation of JNK and p38 pathways. Then the tumor suppressor p53 is activated to transactivate promoters of Bak and p21, and the cell growth is ultimately inhibited. However, this conjecture needs to be further verified.

Taken together, in this study, we demonstrate a novel molecular mechanism through which EGR3 inhibits HCC cell growth. Our study provides new insight into the close cooperation between EGR3 and FasL in tumor regulation, suggesting that EGR3 may be a candidate gene for therapy of HCC.

\section{Acknowledgements}

This study was supported by the National Natural Science Foundation of China (no. 81302112 and no. 81670554) and Wuhan application basis research project (no. 2014060101010052).

\section{References}

1. Yang JD and Roberts LR: Hepatocellular carcinoma: A global view. Nat Rev Gastroenterol Hepatol 7: 448-458, 2010.

2. Huo X, Zhang Q, Liu AM, Tang C, Gong Y, Bian J, Luk JM, Xu Z and Chen J: Overexpression of Yes-associated protein confers doxorubicin resistance in hepatocellullar carcinoma. Oncol Rep 29: 840-846, 2013

3. Tang ZY: Hepatocellular carcinoma - cause, treatment and metastasis. World J Gastroenterol 7: 445-454, 2001.

4. Wu CS, Yen CJ, Chou RH, Li ST, Huang WC, Ren CT, Wu CY and Yu YL: Cancer-associated carbohydrate antigens as potential biomarkers for hepatocellular carcinoma. PLoS One 7: e39466, 2012.

5. Gómez-Martín D, Díaz-Zamudio M, Galindo-Campos M and Alcocer-Varela J: Early growth response transcription factors and the modulation of immune response: Implications towards autoimmunity. Autoimmun Rev 9: 454-458, 2010.
6. Thiel G and Cibelli G: Regulation of life and death by the zinc finger transcription factor Egr-1. J Cell Physiol 193: 287-292, 2002.

7. Kim HJ, Hong JM, Yoon KA, Kim N, Cho DW, Choi JY, Lee IK and Kim SY: Early growth response 2 negatively modulates osteoclast differentiation through upregulation of Id helix-loophelix proteins. Bone 51: 643-650, 2012.

8. Bhattacharyya S, Fang F, Tourtellotte W and Varga J: Egr-1: New conductor for the tissue repair orchestra directs harmony (regeneration) or cacophony (fibrosis). J Pathol 229: 286-297, 2013.

9. Bolli N, Avet-Loiseau H, Wedge DC, Van Loo P, Alexandrov LB, Martincorena I, Dawson KJ, Iorio F, Nik-Zainal S, Bignell GR, et al: Heterogeneity of genomic evolution and mutational profiles in multiple myeloma. Nat Commun 5: 2997, 2014.

10. Stoddart A, Fernald AA, Wang J, Davis EM, Karrison T, Anastasi J and Le Beau MM: Haploinsufficiency of del $(5 q)$ genes, Egr1 and Apc, cooperate with Tp53 loss to induce acute myeloid leukemia in mice. Blood 123: 1069-1078, 2014.

11. Boone DN, Qi Y, Li Z and Hann SR: Egrl mediates p53-independent c-Myc-induced apoptosis via a noncanonical ARF-dependent transcriptional mechanism. Proc Natl Acad Sci USA 108: 632-637, 2011.

12. Wirth M, Stojanovic N, Christian J, Paul MC, Stauber RH, Schmid RM, Häcker G, Krämer OH, Saur D and Schneider G: MYC and EGR1 synergize to trigger tumor cell death by controlling NOXA and BIM transcription upon treatment with the proteasome inhibitor bortezomib. Nucleic Acids Res 42: 10433-10447, 2014.

13. Nishimura Y, Takizawa R, Koike S, Kinoshita A, Satomura Y, Kawasaki S, Yamasue H, Tochigi M, Kakiuchi C, Sasaki T, et al: Association of decreased prefrontal hemodynamic response during a verbal fluency task with EGR3 gene polymorphism in patients with schizophrenia and in healthy individuals. Neuroimage 85: 527-534, 2014.

14. Safford M, Collins S, Lutz MA, Allen A, Huang CT, Kowalski J, Blackford A, Horton MR, Drake C, Schwartz RH, et al: Egr-2 and Egr-3 are negative regulators of T cell activation. Nat Immunol 6: 472-480, 2005

15. Li S, Miao T, Sebastian M, Bhullar P, Ghaffari E, Liu M, Symonds AL and Wang P: The transcription factors Egr2 and Egr3 are essential for the control of inflammation and antigen-induced proliferation of B and T cells. Immunity 37: 685-696, 2012.

16. Liu D, Evans I, Britton G and Zachary I: The zinc-finger transcription factor, early growth response 3 , mediates VEGFinduced angiogenesis. Oncogene 27: 2989-2998, 2008.

17. Baron VT, Pio R, Jia Z and Mercola D: Early growth response 3 regulates genes of inflammation and directly activates IL6 and IL8 expression in prostate cancer. Br J Cancer 112: 755-764, 2015.

18. Suzuki T, Inoue A, Miki Y, Moriya T, Akahira J, Ishida T, Hirakawa H, Yamaguchi Y, Hayashi S and Sasano H: Early growth responsive gene 3 in human breast carcinoma: A regulator of estrogen-meditated invasion and a potent prognostic factor. Endocr Relat Cancer 14: 279-292, 2007.

19. Pio R, Jia Z, Baron VT and Mercola D; UCI NCI SPECS Consortium of the Strategic Partners for the Evaluation of Cancer Signatures-Prostate Cancer: Early growth response 3 (Egr3) is highly over-expressed in non-relapsing prostate cancer but not in relapsing prostate cancer. PLoS One 8: e54096, 2013.

20. Salotti J, Sakchaisri K, Tourtellotte WG and Johnson PF: An Arf-Egr-C/EBP $\beta$ pathway linked to ras-induced senescence and cancer. Mol Cell Biol 35: 866-883, 2015.

21. Cheng H, Hao S, Liu Y, Pang Y, Ma S, Dong F, Xu J, Zheng G, Li S, Yuan W, et al: Leukemic marrow infiltration reveals a novel role for Egr3 as a potent inhibitor of normal hematopoietic stem cell proliferation. Blood 126: 1302-1313, 2015.

22. Liao F, Ji MY, Shen L, Qiu S, Guo XF and Dong WG: Decreased EGR3 expression is related to poor prognosis in patients with gastric cancer. J Mol Histol 44: 463-468, 2013.

23. Suda T, Takahashi T, Golstein P and Nagata S: Molecular cloning and expression of the Fas ligand, a novel member of the tumor necrosis factor family. Cell 75: 1169-1178, 1993.

24. Lavrik IN and Krammer PH: Regulation of CD95/Fas signaling at the DISC. Cell Death Differ 19: 36-41, 2012.

25. Kober AM, Legewie S, Pforr C, Fricker N, Eils R, Krammer PH and Lavrik IN: Caspase-8 activity has an essential role in CD95/ Fas-mediated MAPK activation. Cell Death Dis 2: e212, 2011.

26. Suda T, Okazaki T, Naito Y, Yokota T, Arai N, Ozaki S, Nakao K and Nagata S: Expression of the Fas ligand in cells of T cell lineage. J Immunol 154: 3806-3813, 1995. 
27. Montel AH, Bochan MR, Hobbs JA, Lynch DH and Brahmi Z Fas involvement in cytotoxicity mediated by human NK cells. Cell Immunol 166: 236-246, 1995.

28. Griffith TS, Brunner T, Fletcher SM, Green DR and Ferguson TA: Fas ligand-induced apoptosis as a mechanism of immune privilege. Science 270: 1189-1192, 1995.

29. Uckan D, Steele A, Cherry, Wang BY, Chamizo W, Koutsonikolis A, Gilbert-Barness E and Good RA: Trophoblasts express Fas ligand: A proposed mechanism for immune privilege in placenta and maternal invasion. Mol Hum Reprod 3: 655-662, 1997.

30. Reichmann E: The biological role of the Fas/FasL system during tumor formation and progression. Semin Cancer Biol 12: 309-315, 2002.

31. Zheng SY, Li DC, Zhang ZD, Zhao J and Ge JF: Adenovirusmediated FasL gene transfer into human gastric carcinoma. World J Gastroenterol 11: 3446-3450, 2005.

32. Siena L, Pace E, Ferraro M, Di Sano C, Melis M, Profita M, Spatafora $\mathbf{M}$ and Gjomarkaj M: Gemcitabine sensitizes lung cancer cells to Fas/FasL system-mediated killing. Immunology 141: 242-255, 2014.

33. Bianco SR, Sun J, Fosmire SP, Hance K, Padilla ML, Ritt MG, Getzy DM, Duke RC, Withrow SJ, Lana S, et al: Enhancing antimelanoma immune responses through apoptosis. Cancer Gene Ther 10: 726-736, 2003.

34. Kavurma MM and Khachigian LM: Signaling and transcriptional control of Fas ligand gene expression. Cell Death Differ 10: 36-44, 2003.

35. Mittelstadt PR and Ashwell JD: Cyclosporin A-sensitive transcription factor Egr-3 regulates Fas ligand expression. Mol Cell Biol 18: 3744-3751, 1998.

36. Inoue A, Omoto Y, Yamaguchi Y, Kiyama R and Hayashi SI: Transcription factor EGR3 is involved in the estrogen-signaling pathway in breast cancer cells. J Mol Endocrinol 32: 649-661, 2004.

37. Lee JS and Thorgeirsson SS: Genome-scale profiling of gene expression in hepatocellular carcinoma: Classification, survival prediction, and identification of therapeutic targets. Gastroenterology 127 (Suppl 1): S51-S55, 2004.

38. Thorgeirsson SS, Lee JS and Grisham JW: Molecular prognostication of liver cancer: End of the beginning. J Hepatol 44: 798-805, 2006

39. Tang D, Lotze MT, Kang R and Zeh HJ: Apoptosis promotes early tumorigenesis. Oncogene 30: 1851-1854, 2011.
40. Wang WZ, Li L, Liu MY, Jin XB, Mao JW, Pu QH, Meng MJ, Chen XG and Zhu JY: Curcumin induces FasL-related apoptosis through p38 activation in human hepatocellular carcinoma Huh7 cells. Life Sci 92: 352-358, 2013.

41. Nakamura M, Nagano H, Sakon M, Yamamoto T, Ota H, Wada H, Damdinsuren B, Noda T, Marubashi S, Miyamoto A, et al: Role of the Fas/FasL pathway in combination therapy with interferonalpha and fluorouracil against hepatocellular carcinoma in vitro. J Hepatol 46: 77-88, 2007.

42. El Ojeimy S, McKillop JC, El-Zawahry AM, Holman DH, Liu X, Schwartz DA, Day TA, Dong JY and Norris JS: FasL gene therapy: A new therapeutic modality for head and neck cancer. Cancer Gene Ther 13: 739-745, 2006.

43. Abbas T and Dutta A: p21 in cancer: Intricate networks and multiple activities. Nat Rev Cancer 9: 400-414, 2009.

44. Westphal D, Dewson G, Czabotar PE and Kluck RM: Molecular biology of Bax and Bak activation and action. Biochim Biophys Acta 1813: 521-531, 2011.

45. Swindall AF and Bellis SL: Sialylation of the Fas death receptor by ST6Gal-I provides protection against Fas-mediated apoptosis in colon carcinoma cells. J Biol Chem 286: 2298222990, 2011.

46. Yang X, Khosravi-Far R, Chang HY and Baltimore D: Daxx, a novel Fas-binding protein that activates JNK and apoptosis. Cell 89: 1067-1076, 1997.

47. Juo P, Kuo CJ, Reynolds SE, Konz RF, Raingeaud J, Davis RJ, Biemann HP and Blenis J: Fas activation of the p38 mitogenactivated protein kinase signalling pathway requires ICE/CED-3 family proteases. Mol Cell Biol 17: 24-35, 1997.

48. Lee KB, Kim KR, Huh TL and Lee YM: Proton induces apoptosis of hypoxic tumor cells by the p53-dependent and p38/JNK MAPK signaling pathways. Int J Oncol 33: 1247-1256, 2008.

49. Maiuri MC, Galluzzi L, Morselli E, Kepp O, Malik SA and Kroemer G: Autophagy regulation by p53. Curr Opin Cell Biol 22: $181-185,2010$

50. Gartel AL and Tyner AL: The role of the cyclin-dependent kinase inhibitor p21 in apoptosis. Mol Cancer Ther 1: 639-649, 2002.

51. Graupner V, Alexander E, Overkamp T, Rothfuss O, De Laurenzi V, Gillissen BF, Daniel PT, Schulze-Osthoff K and Essmann F: Differential regulation of the proapoptotic multidomain protein Bak by p53 and p73 at the promoter level. Cell Death Differ 18: 1130-1139, 2011. 